\title{
Potential implications and advantages of implementing protected u-turns in Lahore, Pakistan
}

\author{
Muhammad Tanveer ${ }^{1}{ }^{*}$, Huapu Lu ${ }^{2}$, Faizan Ahmad Kashmiri ${ }^{3}$, Salman Maqbool ${ }^{4}$ \\ ${ }_{1,2}$ Department of Civil Engineering, Institute of Transportation Engineering, Tsinghua University, Beijing, China \\ ${ }^{3,4}$ National Engineering Services Pakistan (NESPAK), Lahore, Pakistan
}

\section{Keywords \\ Protected \\ U-turns \\ Pedestrians \\ Geometric \\ Design \\ Advantages \\ Disadvantages \\ Implications}

Received: 5 October 2018

Accepted: 9 November 2018

Published: 19 December 2018

\begin{abstract}
Multiple interventions and innovations in geometric layouts on the road reduce travel time, reduce traffic congestion, and increase mobility flow. One of these improvements is the introduction of protected U-turns at junctions. With the implementation of this technique, the through-moving traffic is given priority as they traverse without any obstruction. Such a system is beneficial if the designer aims to design the junction signal-free and it lessens the travel time to some extent. Although it is practical, certain implications and consequences arise with the execution of such an unconventional layout. Pakistan currently utilizes this idea in designing its roads currently. This paper aims to highlight the potential consequences as well as the advantages of this arrangement. To better understand these phenomena, some existing examples are given of Lahore in this paper to pinpoint the pressing issues at large, i.e., obstructions to pedestrians, land acquisition issues in urban areas, need to provide pedestrian bridges which ultimately increase the cost of the project. The contribution of this research will aid the geometric designers and traffic engineers to take multiple considerations in the planning/designing the stage from all ends before instigating this pattern on roads.
\end{abstract}

\section{INTRODUCTION}

Traffic congestion is increasing day by day. The conventional signalized and unsignalized intersections sometimes provide a major delay, accidents and an increase in travel time. In order to counter this, geometric interventions are typically introduced. One of these interventions is the usage of a protected U-turn. Left turns in this type of intersection are banned by rerouting and providing a protected raised U-turn ahead. The vehicles traverse this new route and subsequently make a right turn in the end when they come back to the intersection. Several studies under limited scope and constraints have been carried out to study the impact of $U$-Turns on signalized and unsignalized intersection $[1,2,3,4,5,6,7]$. The goal of this paper to highlight the impact of U-turns in terms of advantages and disadvantages by studying some of the examples of Lahore, Pakistan as they are currently used by various organizations to make the through movement signal free.

\section{LITERATURE REVIEW}

The travel efficiency of unconventional alternatives in comparison to conventional intersections under different volume-based scenarios was analyzed [8]. The advantages and disadvantages of 3 different unconventional left turn alternatives for urban and sub-urban arterials were studied [9]. Median U-Turn Crossover Design (MUT), Super Street Median Cross over design (SSM) and Two Way Left Turn Lanes (TWLTL) through CORSIM were analyzed and indicated that both SSM and MUT geometric interventions improved travel time and average speed in comparison to TWLTL $[10,11,12]$. Similarly, analysis of seven unconventional intersections through CORSIM was done and it was shown that at least one of the unconventional intersections had the lowest travel time [13]. Furthermore, the quad-

\footnotetext{
*Corresponding author: Muhammad Tanveer

†email: qgw14@mails.tsinghua.edu.cn
} 
rant road intersection and median-turn designs strived for the lowest average time in the study [13]. Similarly, it was studied that safety was greater for right turns followed by U-turns than for direct left turns [14]. It was indicated that indirect left turns could improve safety when accompanied by restricted left turns at nearby unsignalized intersections [15]. Moreover, it was showed that upstream signalized crossover could reduce average vehicle delays in comparison to a conventional intersection though VISSIM simulation [16]. Three different cases of super street design with conventional intersection design were compared. Simulation results showed that the super street design with one U-turn lane outperforms the comparable conventional intersection under high traffic volumes [17]. Two unconventional intersections i.e. cross over displaced left turn and upstream signalized crossover intersection was compared with conventional intersections though VISSIM Simulation. They showed that both outdone the conventional intersection under different volumes scenarios [18]. In another study the operational performance of three unconventional intersections was compared and evaluated: Continuous Flow Intersection (CFI), Parallel Flow Intersection (PFI) and Upstream signalized Crossover (USC) with a conventional intersection through VISSIM Simulations. The results showed that all three unconventional intersections outperformed conventional intersection [19]. A procedure was developed to help transportation professionals evaluate the impacts of proposed indirect driveway left-turn treatments on traffic operations at downstream signalized intersections [20]. Their research developed a negativebinomial model developed for predicting U-turn volume on a left-turn approach at a signalized intersection during weekday peak periods. They concluded that with the estimated U-turn volume, designers could also make various design decisions regarding the left-turn bay where Uturns are provided [20]. Unconventional design alternatives such as raised U-turn facility showed improvement over the conventional intersection in terms of travel times [21]. However, right-of-way, traffic safety strategies and measure, road signing, pavement marking, pedestrian facilities and access were not part of their study.

\section{PROTECTED U-TURNS IN LAHORE}

The second largest metropolitan city of Pakistan and the capital of the province Punjab is Lahore with an area of 1172 square kilometers. The population of Lahore was 11126000 persons in 2017. The percentage of the population has increased by about 76 percent from 1998 to 2017 and the density person per square kilometer is around 6279 [22]. The U-turns presented in this study were first studied in terms of travel time [21].

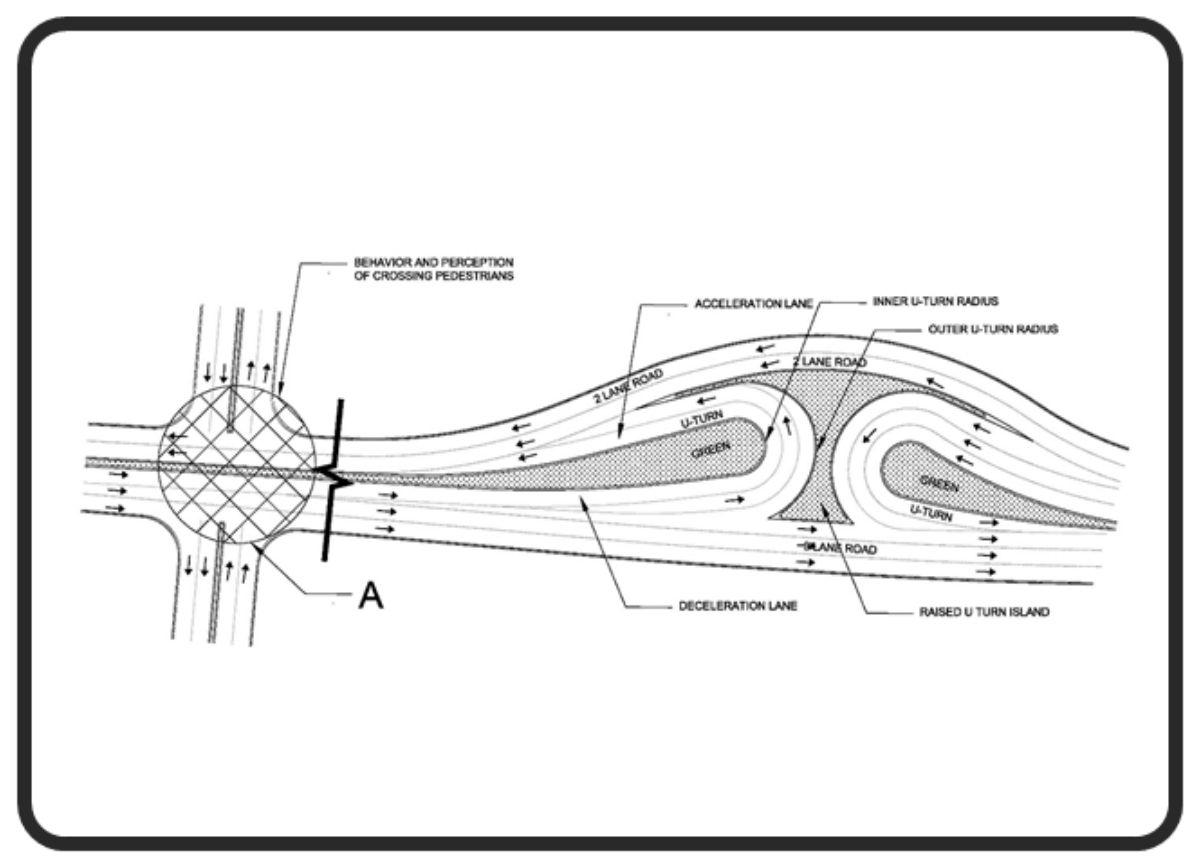

Fig. 1. Typical u-turn Facility by rerouting left turn 
As the above figure depicts, there is no need to provide a signal for motorists of any direction as per the previous studies as this U-turn provides an uninterrupted traffic flow without the usage of signals. A second U-turn facility is provided behind " $\mathrm{A}$ " intersection. With the usage of both intersections, no signal is needed. This type of U-turn is widely used in Pakistan and the Middle East as a means to ease the congestion on a signalized intersection. In some cases, in Pakistan for the roundabout, which has high right turning volumes (Pakistan is Left Hand Traffic Drive), the right turning is also banned and motorists have to make a left turn followed by U-turn to reach their destination. This is done to ease the congestion on a roundabout because the right turning volume is very high in this scenario. The main elements of this U-turn are the following:

i. Acceleration Lanes

ii. Deceleration Lanes

iii. Inner U-turn Radius

iv. Outer U-turn Radius

v. Raised U-turn Island

The median is bulged out to increase the inner U-turn ra- dius typically 2-6 meters. The outer U-turn radius should be adequate enough to provide an uninterrupted U-turn maneuver. This depends on the type of vehicle this U-turn is being designed for as per "A Policy on Geometric Design of Highways and Streets, American Association of State Highway and Transportation Officials, AASHTO 2011" [23] guidelines i.e., Passenger Car, WB-40 etc. The geometric design of such facility is typically location dependent and may vary as per the standards set by "A Policy on Geometric Design of Highways and Streets, American Association of State Highway and Transportation Officials, AASHTO) 2011" [23]. Thus, the typical U-turn shown in Figure 1 is used in various locations in the city of Lahore to make the through movement signal free in congested areas. Now let us consider the case of an existing junction which utilizes the concept of these U-turns. In some cases, in Pakistan for the roundabout, which has high right turning volumes (Pakistan is Left Hand Traffic Drive), the right turning is also banned and motorists have to make a left turn followed by U-turn to reach their destination as shown in Figure 2.

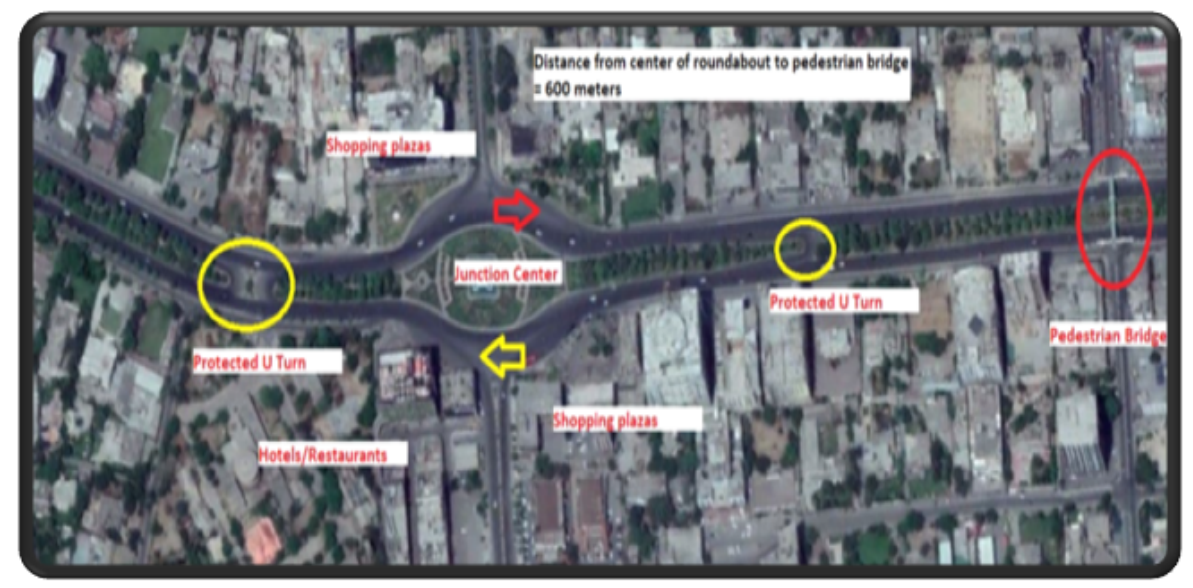

Fig. 2. Applications of U turn at a typical junction (Liberty Junction, Lahore)

This is done to ease the congestion on a roundabout because the right turning volume is very high in this scenario. The colored arrows (Red and Yellow) in Figure 2 shows the direction of traffic in this unconventional roundabout located in Pakistan. The traffic turns with the help of U-turn facilities. This junction is quite famous in the city as it is surrounded by commercial and residential surroundings. Despite easing the congestion for motorists, the crossing pedestrians are suffering the most here because the through traffic is moving at a high speed which causes the pedestrians to accept shorter gap and maneuver at a high crossing speed as they are vulnerable to accidents. Since the through movement is given priority here, a need for an elevated structure (pedestrian bridge) is necessary to provide safety and maneuverability for pedestrians. There is an only a pedestrian bridge in the area and it almost 600 meters (near Hafeez Centre Market) from the center of the junction. The road users could not cross near junction even if they wanted to due to high speed through movements. This problem is high with elderly persons and individuals with disabilities who suffer the most in these types of scenarios. A plausible suggestion would be to provide an additional pedestrian bridge near the center of the junction. The provision of U-turns has been immensely beneficial for this 
junction in terms of road mobility but the considerations to pedestrian crossings should be considered also. Pedestrians might use a "rolling gap" to cross the street if the lanes are not clear and traffic volume is high [24]. The pedestrians anticipated that the lanes would become clear as they crossed the street [24].

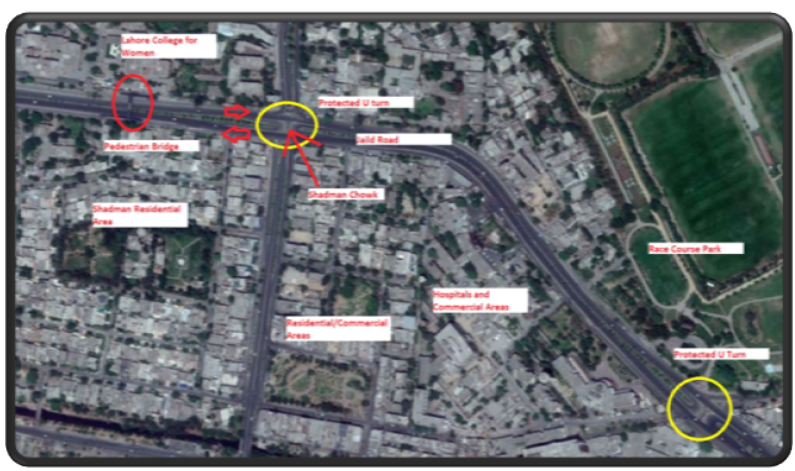

Fig. 3. Applications of U turn at a typical junction (Shadman Junction, Lahore)

Similarly, there is another example shown in Figure 3. The location is Shadman Junction which is one of the most vital junctions in Lahore surrounded by commercial, educational and residential areas. There is only one pedestrian bridge in that area and that is near the Lahore College for
Women (mainly used by students in peak time). The bridge was made even before these unconventional interventions to make the Jail Road signal free. Apart from that, there is no other crossing facility. Since there are hospitals and commercial areas nearby so crossing roads is essential. However, this issue has not been taken into consideration by the pertinent authorities. The pedestrians than are forced to cross roads at grade. A street simulation was compared between young and elder pedestrians. The elder pedestrians received 6 months of training and showed significant improvement in their mobility and behavior [25]. Despite their improvement, their ability to judge the oncoming car's speed remained did not improve and they were found to make more risks as the car's speed increased [25]. Their findings suggested that effective speed reduction measures should be introduced for safe crossings of the elderly [25]. A study was regarding conducted on overpasses instead of signalized intersections and mid-block crossings. The study suggested that pedestrian preferred to cross at street level [26]. The condition of the overpass, overpass length, time spent in overpass vs the time spent on the street crossing, the presence of escalators/elevators affect the overpass usage [26].

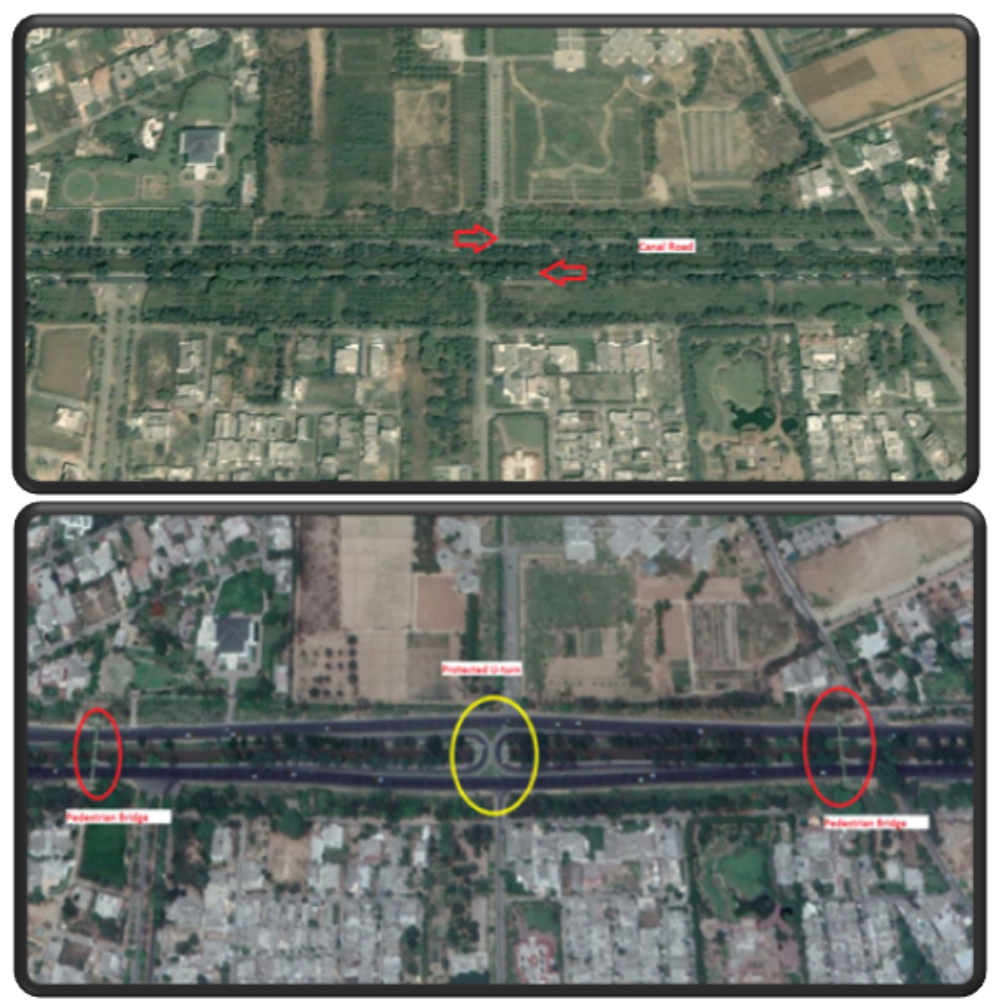

Fig. 4.4 (a) Canal Road in 2005 (b) Canal Road in 2019, Applications of U turn at a typical junction (Canal Road, Lahore) 
Figure 4 shows the aerial view of the location of canal road, Lahore in 2005 and in 2019. Canal Road is the main arterial road of the city interconnecting the secondary and tertiary links. Usually, before the implementation of protected U-turns, people had to maneuver U-turn behavior at crossing junctions at Canal Road thus increasing travel time and queue lengths at the junctions but with the implementation of these protected U-turns, the ease in mobility and overall traffic flow has improved significantly. Moreover, two pedestrian bridges are provided at the start and end of the protected U-turn for the safe crossing of pedestrians. This is one of the prime examples of these U-turns operating as per expected results without disturbing the crossroad behaviors. However, it is evident that most of the green trees were removed to provide the U-turn thus damaging the environment and leading to a pollutant atmosphere. The removal of the trees also leads to discomfort of the driver due to lights flashing into the eyes of the driver from opposing side traffic. Land acquisition was also mad on both sides of the road to accommodate the bulging part of the U-turn thus increasing the cost of the project. This is one of the major drawbacks of these U-turns that in an urban congested and densely covered area, land acquisition is an essential phase which increases the overall cost of the project, thus limiting its usage in several design considerations.

\section{CONCLUSION}

With increasing traffic congestion and travel demand and budget issues of new roads, traffic and highway engineers develop several unconventional designs to improve the overall traffic flows of transportation systems. The typical example of protected U-turns discussed in this case is also one of those layouts. Although it is practical and it reduces the travel time up to an extent, it also has some several implicit drawbacks that come alongside its implementation. It is recommended to study the responses of pedestrians and drivers to develop key strategies which can enhance the safety of pedestrians and overall reduce the travel time in future studies. For example, in case of pedestrians how many times they travel at this location, how often do they cross form designated or non-designated sidewalk, if the existing road safety facilities are safe for manoeuvring, do they think signage or their phase timing should be provided, should $U$ turn be close to pedestrian crossings and jucntions? Do you feel safe in the presence of the unconventional $U$ turn as shown in Figure 1, what kind of safety measures should be provided as per your experience etc. An in depth study in this topic will aid the designers and planners to consider various alternatives before adopting this strategy.

\section{ACKNOWLEDGMENT}

This research was funded by the "Research of Transportation Strategy for Powerful Nation" (ID: 2017ZD07), the major consulting project of the Chinese Academy of Engineering. and thanks to Dr. Ruimin Li's suggestion.

\section{REFERENCES}

[1] J. C. Adams and J. E. Hummer, "Effects of u-turns on left-turn saturation flow rates," Transportation Research Record, vol. 1398, pp. 90-100, 1993.

[2] P. Liu, J. J. Lu, J. Fan, J. C. Pernia, and G. Sokolow, "Effects of u-turns on capacities of signalized intersections," Transportation Research Record, vol. 1920, no. 1, pp. 74-80, 2005. doi: https://doi.org/10.3141/1920-09

[3] P. Liu, J. Lu, G. Sokolow, and H. Zhou, "Operational effects of u-turns as alternatives to direct left-turns," Journal of Transportation Engineering, vol. 133, no. 5, p. 327-334, 2007. doi: https://doi.org/10.1061/(asce)0733-947x(2007) 133:5(327)

[4] A. Pirdavani, T. Brijs, T. Bellemans, G. Wets, and K. Vanhoof, "Travel time evaluation of an innovative u-turn facility on urban arterial roadways," in Proceeding of 87th Annual Meeting of the Transportation Research Board, Tokyo, Japan, 2009.

[5] P. Liu, J. J. Lu, F. Hu, and G. Sokolow, "Capacity of u-turn movement at median openings on multilane highways," Journal of Transportation Engineering, vol. 134, no. 4, pp. 147-154, 2008. doi: https://doi.org/10.1061/(asce) 0733-947x(2008)134:4(147)

[6] S. N. Cubero, S. McLernon, and A. Sharpe, "Over-speeding warning system using wireless communications for road signs and vehicles," Journal of Advances in Technology and Engineering Studies, vol. 2, no. 5, pp. 140-155, 2016. doi: https://doi.org/10.20474/jater-2.5.2

[7] P. Wongsai and W. Pawgasame, "Analysis of a crime scene getaway vehicle's escaping path," International Journal of Technology and Engineering Studies, vol. 2, no. 5, pp. 134-139, 2016. 
[8] J. Boone and J. Hummer, "Unconventional design and operation strategies for over-saturated suburban arterials," North Carolina Department of Transportation, Raleigh, NC, Technical report, 1994.

[9] J. E. Hummer, "Unconventional left-turn alternatives for urban and suburban arterials—part two," ITE Journal, vol. 68, no. 11, pp. 101-106, 1998.

[10] J. D. Reid and J. E. Hummer, "Analyzing system travel time in arterial corridors with unconventional designs using microscopic simulation," Transportation Research Record, vol. 1678, no. 1, pp. 208-215, 1999. doi: https://doi.org/10. 3141/1678-25

[11] Y. L. Yeh and C. H. Chiang, "Sequence behavior of vortical development and evolution of a low-speed rectangular jet," Journal of Advances in Technology and Engineering Research, vol. 3, no. 4, pp. 144-149, 2017. doi: https://doi.org/10. 20474/jater-3.4.4

[12] J. Joonwoo, K. Dong-Hyun, C. Bumsuk, K. Geonwoo, and K. Yoo-Sung, “A construction of vehicle image and ground truth database for developing vehicle maker and model recognitions," International Journal of Technology and Engineering Studies, vol. 3, no. 6, pp. 229-235, 2017. doi: https://doi.org/10.20469/ijtes.3.40002-6

[13] J. D. Reid and J. E. Hummer, "Travel time comparisons between seven unconventional arterial intersection designs," Transportation Research Record, vol. 1751, no. 1, pp. 56-66, 2001. doi: https://doi.org/10.3141/1751-07

[14] J. Lu and L. X. Dissanayake, "Safety evaluation of right turns followed by u-turns as an alternative to direct left turns-conflict analysis," 2001. [Online]. Available: https://urlzs.com/bvpC8

[15] I. B. Potts, Safety of U-Turns at unsignalized median openings. Florida, FL: Transportation Research Board, 2004, vol. 524Fl.

[16] V. Tabernero and T. Sayed, "Upstream signalized crossover intersection: An unconventional intersection scheme," Journal of Transportation Engineering, vol. 132, no. 11, pp. 907-911, 2006. doi: https://doi.org/10.3141/1961-06

[17] T. Kim, P. K. Edara, and J. G. Bared, "Operational and safety performance of a nontraditional intersection design: The superstreet," in Proceeding of 86th Annual Meeting of the Transportation Research Board, New York, NY, 2007.

[18] M. E. Esawey and T. Sayed, "Comparison of two unconventional intersection schemes: Crossover displaced left-turn and upstream signalized crossover intersections," Transportation Research Record, vol. 2023, no. 1, pp. 10-19, 2007. doi: https://doi.org/10.3141/2023-02

[19] S. Cheong, S. Rahwanji, and G.-L. Chang, "Comparison of three unconventional arterial intersection designs: Continuous flow intersection, parallel flow intersection, and upstream signalized crossover," in Proceeding of the 52 11th International IEEE Conference on Intelligent Transportation System, Beijing, China, 2008.

[20] T. Guo, P. Liu, J. J. Lu, L. Lu, and B. Cao, "Procedure for evaluating the impacts of indirect driveway left-turn treatments on traffic operations at signalized intersections," Journal of Transportation Engineering, vol. 137, no. 11, pp. 760-766, 2011. doi: https://doi.org/10.1061/(asce)te.1943-5436.0000258

[21] A. Pirdavani, T. Brijs, T. Bellemans, and G. Wets, "Travel time evaluation of a u-turn facility: Comparison with a conventional signalized intersection," Transportation Research Record, vol. 2223, no. 1, pp. 26-33, 2011. doi: https: //doi.org/10.3141/2223-04

[22] Bureau of Statistics Government of the Punjab Lahore, "Punjab development statistic," 2017. [Online]. Available: https://urlzs.com/UhSkc

[23] American Association of State Highway and Transportation, "A policy on geometric design of highways and streets," 2011. [Online]. Available: https://urlzs.com/z37mQ

[24] M. A. Brewer, K. Fitzpatrick, J. A. Whitacre, and D. Lord, "Exploration of pedestrian gap-acceptance behavior at selected locations," Transportation Research Record, vol. 1982, no. 1, pp. 132-140, 2006. doi: https://doi.org/10.3141/1982-18

[25] A. Dommes, V. Cavallo, F. Vienne, and I. Aillerie, "Age-related differences in street-crossing safety before and after training of older pedestrians," Accident Analysis \& Prevention, vol. 44, no. 1, pp. 42-47, 2012. doi: https://doi.org/10. 1016/j.aap.2010.12.012

[26] Y. Alver and P. Onelcin, "Gap acceptance of pedestrians at overpass locations," Transportation Research Part F: Traffic Psychology and Behaviour, vol. 56, pp. 436-443, 2018. doi: https://doi.org/10.1016/j.trf.2018.05.010 